\title{
Optical switching in metal-slit arrays on nonlinear dielectric substrates
}

\author{
S. Carretero-Palacios, ${ }^{1, *}$ Alexander Minovich, ${ }^{2}$ Dragomir N. Neshev, ${ }^{2}$ \\ Yuri S. Kivshar, ${ }^{2}$ F. J. Garcia-Vidal, ${ }^{3}$ L. Martin-Moreno, ${ }^{1}$ and Sergio G. Rodrigo ${ }^{1}$ \\ ${ }^{1}$ Instituto de Ciencia de Materiales de Aragon and Departamento de Fisica de la Materia Condensada, \\ CSIC-Universidad de Zaragoza, E-50009, Zaragoza, Spain \\ ${ }^{2}$ Nonlinear Physics Centre, Research School of Physics and Engineering, Australian National University, Canberra, Australia \\ ${ }^{3}$ Departamento de Fisica Teorica de la Materia Condensada, Universidad Autonoma de Madrid, Madrid 28049, Spain \\ ${ }^{*}$ Corresponding author: sol@unizar.es
}

Received October 29, 2010; revised November 1, 2010; accepted November 10, 2010; posted November 29, 2010 (Doc. ID 129143); published December 15, 2010

\begin{abstract}
We propose a scheme for an optical limiter and switch of the transmitted light intensity in an array of subwavelength metallic slits placed on a nonlinear Kerr-type dielectric substrate of finite thickness, where the geometrical parameters are designed for operation at telecom wavelengths. Our approach is based on the abrupt changes of the output light intensity observed in these systems near transmission minima. (C) 2010 Optical Society of America OCIS codes: $240.6680,190.5940,250.5403$.
\end{abstract}

Nanostructuring of metal surfaces appears exceptionally attractive for novel nonlinear photonic applications due to a strong enhancement of the electromagnetic (EM) field near corrugated surfaces. Prominent examples include surface-enhanced Raman scattering [1] and plasmon-enhanced high-harmonic generation [2]. However, the drawback of using metallic elements is the introduction of strong loss. Therefore, when metals are involved, only schemes utilizing short device lengths can be tolerated in practice to observe noticeable nonlinear phenomena, while any schemes relying on phase accumulation are intrinsically excluded.

A proposed platform for enhanced nonlinear optical manipulation is the array of subwavelength apertures in metal films [3,4]. Nonlinear properties of subwavelength metallic apertures in combination with nonlinear dielectrics have been studied for their potential uses in enhanced nonlinear beam manipulation $[5,6]$, optical bistability $[7,8]$, and switching $[9,10]$. Such geometries rely on the phenomenon of enhanced optical transmission [11-13] associated with distinct resonances in the linear transmission spectrum.

Here we present a proposal for (i) an optical limiter (OL) of the transmitted intensity, in which the output intensity decreases when the incident one increases, and (ii) an optical switch (OS), where the output intensity increases abruptly under a small change of the incident power. These two operating modes are found in an array of metallic slits placed on a nonlinear Kerr-type dielectric layer, at the telecom regime. The inset of Fig. 1 shows schematically the structure analyzed, as well as the direction of illumination considered. We assume air at the illuminated and transmission regions, and also inside the slits.

We study the nonlinear optical response at a fixed wavelength close to the transmission minimum [see Fig. 1 (top)], where sharp variations in the spectrum take place within a short wavelength range. We assume nonlinear response only in the dielectric layer, while the nonlinear susceptibility in the metal [14] is neglected. In the MKS system of units, the optical response of the dielectric material due to a third-order nonlinear susceptibility is described by the relations of both displacement $(\vec{D})$ and polarization $(\vec{P})$ vectors with the electric field $(\vec{E})[\underline{15}]$ : $\vec{D}=\varepsilon_{0} \vec{E}+\vec{P}$, where $\vec{P} \simeq \varepsilon_{0}\left[\chi^{(1)} \vec{E}+\chi^{(3)}(\vec{E} \cdot \vec{E}) \vec{E}\right]$ and $\varepsilon_{0}$ is the dielectric constant of vacuum. The scalar quantities $\chi^{(1)}$ and $\chi^{(3)}$ represent the linear and third-order nonlinear susceptibilities, respectively. Since the change of the dielectric constant yielded by the local EM field is percentually small, it is common to approximate the refractive index as $n=n_{0}+n_{2} I$, where $I=n_{0} c \varepsilon_{0}|E(\vec{r})|^{2} / 2, n_{0}$ is the linear refractive index, and $n_{2}=\frac{3 \chi^{(3)}}{4 n_{0}^{2} \varepsilon_{0}}[\underline{15}]$ is the Kerr coefficient.

We have employed the finite-difference time-domain method to simulate both the linear [16] and nonlinear optical response of the structure [1] ]. The system is

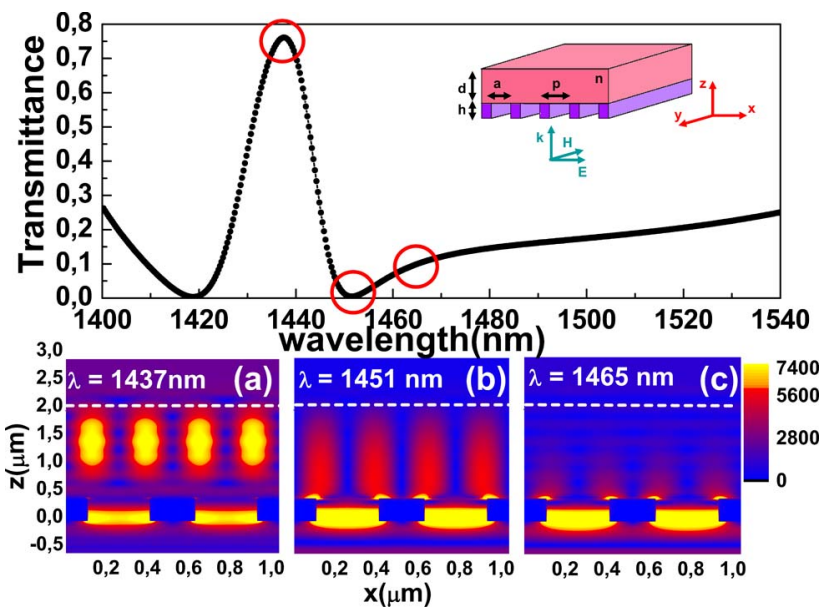

Fig. 1. (Color online) Linear transmittance through a gold slit array $(P=520 \mathrm{~nm}, a=300 \mathrm{~nm}, h=350 \mathrm{~nm})$ on a dielectric substrate of finite thickness $\left(n_{0}=2.8\right.$ and $\left.d=1650 \mathrm{~nm}\right)$. (a)-(c) Modulus of the electric field, $|\vec{E}|$, evaluated at the $x-z$ plane for two unit cells at $\lambda_{\max }=1437 \mathrm{~nm}, \lambda_{\min }=1451 \mathrm{~nm}$, and $\lambda_{1}=1465 \mathrm{~nm}$, respectively. The dashed white lines mark the end of the dielectric layer. 
illuminated with a normal incident $p$-polarized plane wave. Unless otherwise stated, the duration of the pulse is $\tau \sim 2.4 \mathrm{ps}$. This pulse is slowly switched on and off, so it has a smooth Fourier transform (in this case, with spectral bandwidth of $\sim 3 \mathrm{~nm}$ ). We have checked that, under such excitation, the process of third-harmonic generation forms less than $1 \%$ of the total output energy and, therefore, only the optical properties at the fundamental frequency are considered.

The dielectric constant of gold $\left(\varepsilon_{m}\right)$ is obtained from the experimental values tabulated in [18] and fitted to a Drude-Lorentz model [19]. The nonlinear dielectric is assumed to be isotropic, homogeneous, and dispersionless. Furthermore, absorption in the dielectric is neglected in our simulations. The linear refractive index is chosen to be $n_{0}=2.8$, a typical value for materials with large Kerr coefficients, following Miller's rule [15].

To illustrate our proposal, we have chosen the following set of parameters: a $1650 \mathrm{~nm}$ thickness dielectric slab, array period $p=520 \mathrm{~nm}$, metal film thickness $h=350 \mathrm{~nm}$, and slit width $a=300 \mathrm{~nm}$. The precise values of these parameters are arbitrary but are chosen in order to (i) be within the range accessible to experiments and (ii) provide a sharp transmission minimum at near-IR, in this case, $\lambda_{\min }=1451 \mathrm{~nm}$ [Fig. 1 (top)]. The spectral position of this minimum depends on the geometrical parameters in a complex way. Thus, the consideration of other ranges of working wavelengths would require a fine tuning of geometrical parameters (i.e., other $\lambda_{\text {min }}$ ) through computation of the linear transmittance. In Figs. 1(a)-1(c) we plot the modulus of the electric field, $|\vec{E}|$ (evaluated at the $x-z$ plane for two unit cells) for three different wavelengths: $\lambda_{\max }=1437 \mathrm{~nm}, \lambda_{\min }$, and $\lambda_{1}=1465 \mathrm{~nm}$, respectively. For $\lambda_{1}$ and $\lambda_{\min }$, we observe field enhancement inside the slits and around their corners, while, at $\lambda_{\max }$, a guided mode appears inside the dielectric layer.

Next, we study the changes in the transmission through the metal slits with increase of the light intensity for four different wavelengths redshifted compared to $\lambda_{\min }$. We scale both the incident $\left(I^{\text {in }}\right)$ and transmitted ( $\left.I^{\text {out }}\right)$ intensities by $n_{2}$, so our results are valid for different (current or future) nonlinear materials. Also, $n_{2} I^{\text {out }}$ reflects the average change of the refractive index, $\Delta n$, in the dielectric film (we will discuss spatial distribution of $\Delta n$ later on). Figure 2 presents, with solid symbols, the results for $n_{2} I^{\text {out }}$ as a function of $n_{2} I^{\text {in }}$, together with the corresponding linear results $\left(n_{2} I_{\mathrm{Lin}}^{\text {out }}\right)$. As expected, at low input intensities the transmission follows the linear dependence. However, as the intensity is increased, the transmission saturates and then drops. This behavior corresponds to a nonlinear intensity limiter. For higher $I^{\text {in }}$, the transmission exhibits a steep rise, switching to a high-transparency state. This can be heuristically understood by noting that nonlinear effects correspond to an increase of $n$ and that, in the linear regime, the increase of $n$ shifts the transmission spectrum to longer wavelengths. In the OL regime, as the incident intensity increases, the linear transmittance decreases. Eventually, after the minimum transmittance is reached, the output intensity would be boosted by both the increase of the incident intensity and the corresponding increase in linear transmittance, leading to a large increment of $I^{\text {out }}$ within a narrow range of incident intensities.

Clearly, the incident intensities to achieve OL or OS strongly depend on the incident wavelength. The vertical scale in Fig. 2 breaks in the region of $n_{2} I^{\text {out }}=(8-12) \times 10^{-4}$ in order to mark up the features' visibility at low output intensities. In Fig. 2, the switching is seen for two wavelengths, $\lambda_{1}=1465 \mathrm{~nm}$ and $\lambda_{2}=1475 \mathrm{~nm}$, in the range of intensities chosen, with the final $I^{\text {out }}$ being much larger than $I_{\text {Lin }}^{\text {out }}$ Precisely, OL occurs for all considered wavelengths (and also OS, although this is not shown in the figure for the two largest wavelengths), but the input intensities for minimum output increase as the working wavelength separates from $\lambda_{\text {min }}$. Figure 2 also shows that the considered nonlinear effects are still present for shorter pulses ( $\tau \sim 500 \mathrm{fs}$, although the OL is less pronounced and the OS occurs within a wider range of $I^{\text {in }}$ ), which, in real experiments, would reduce the influence of the free carrier absorption or thermal effects.

In Fig. 3, panels (a)-(d) show the local change in the refractive index within the nonlinear dielectric slab (evaluated at the $x-z$ plane for two unit cells) for the structure in Fig. 1 and for four different input intensities at $\lambda_{1}=$ $1465 \mathrm{~nm}^{-}$at a moment just before the plane wave is switched off ( 2.4 ps). The intensity values cover both the linear regime as well as the intensity range when the OL and the OS occur. Importantly, the variation of the refractive index is not uniform and resembles the profiles of the modulus of the electric field in the linear regime [Figs. 1(a)-1(c)] at the related wavelengths.

Let us now discuss possible materials to operate the OL and OS. Chalcogenide glasses, such as $\mathrm{As}_{2} \mathrm{Se}_{3}$, possess $n_{0} \approx 2.8$ and high $n_{2} \approx 1.1 \times 10^{-4} \mathrm{~cm}^{2} / \mathrm{GW}$ [20], featuring low linear and two-photon absorption at IR wavelengths. However, in real experiments, dielectric slabs made of these materials support a maximum change in the refractive index, $\Delta n^{\max } \approx 0.0001$, before being damaged, a threshold much smaller than the change of the refractive index here obtained when metals are involved

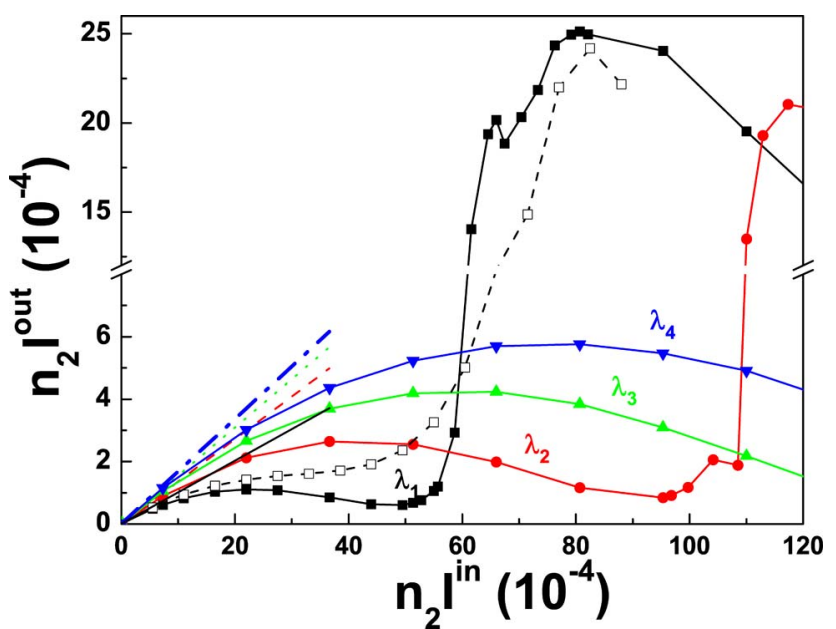

Fig. 2. (Color online) Transmitted intensity versus incident intensity for: solid symbols, $\lambda_{1}=1465 \mathrm{~nm}, \lambda_{2}=1475 \mathrm{~nm}$, $\lambda_{3}=1485 \mathrm{~nm}$, and $\lambda_{4}=1495 \mathrm{~nm}$. Linear output in each case is given by solid, dashed, dotted, and dashed-dotted lines, respectively. Open symbols, $\lambda_{1}=1465 \mathrm{~nm}$, for a short pulse of $\sim 500$ fs duration (spectral bandwidth of $\sim 12 \mathrm{~nm}$ ). 

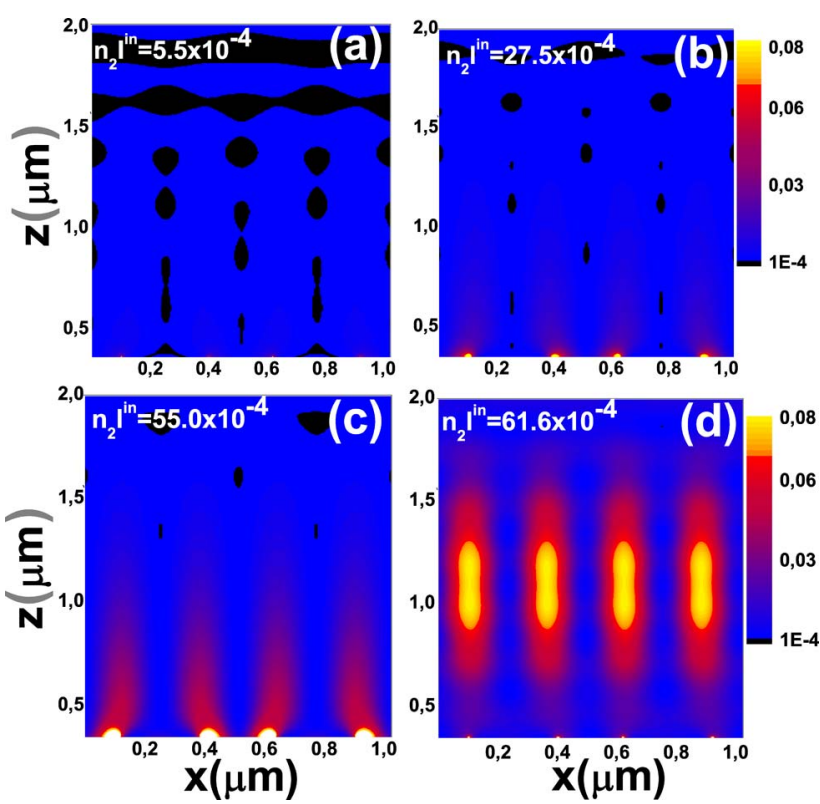

Fig. 3. (Color online) Local change in the refractive index, $\Delta n$, within the dielectric slab for the same structure considered in Fig. 1 at $\lambda_{1}=1465 \mathrm{~nm}$. For different input intensities (a) $n_{2} I^{\text {in }}=5.5 \times 10^{-4}$, (b) $n_{2} I^{\text {in }}=27.5 \times 10^{-4}$, (c) $n_{2} I^{\text {in }}=55 \times$ $10^{-4}$, and (d) $n_{2} I^{\text {in }}=61.6 \times 10^{-4}$.

( $\Delta n^{\max } \approx 0.1$ in some regions of Fig. 3). Nevertheless, semiconductors could appear as better candidates, since they have similar linear and nonlinear refractive indices to that of chalcogenides but support a much higher $\Delta n^{\max } \approx$ 0.1 , as reported in $[21,22]$. In any case, appropriate candidates for real experiments must have both high $\Delta n$ and $n_{2}$ and also must behave as Kerr-type materials with low absorption in a wide range of local intensities.

In conclusion, we have theoretically studied the nonlinear response of a slit array deposited on a dielectric substrate with Kerr nonlinearity. We have described both a nonlinear intensity limiter and an optical switch, at the telecom range. The physical mechanism of both operation modes is based on sharp variations in the linear transmission close to the transmission minima present in arrays of subwavelength apertures in metal films.

We acknowledge the support from the Spanish Ministry of Science and Innovation (the projects MAT200806609-C02 and CSD2007-046-Nanolight.es), the Consejo
Superior de Investigaciones Cientificas (project Intramural 2008601253), and the Australian Research Council.

\section{References}

1. S. Nie and S. R. Emory, Science 275, 1102 (1997).

2. S. Kim, J. H. Jin, Y. J. Kim, I. Y. Park, Y. Kim, and S. W. Kim, Nature 453, 757 (2008).

3. G. A. Wurtz and A. V. Zayats, Laser Photon. Rev. 2, 125 (2008).

4. S. Xie, H. Li, H. Xu, X. Zhou, S. Fu, and J. Wu, Opt. Commun. 283, 998 (2010).

5. C. Min, P. Wang, X. Jiao, Y. Deng, and H. Ming, Opt. Express 15, 9541 (2007).

6. M. A. Vincenti, A. D'Orazio, M. Buncick, N. Akozbek, M. J. Bloemer, and M. Scalora, J. Opt. Soc. Am. B 26, 301 (2009).

7. J. A. Porto, L. Martín-Moreno, and F. J. García-Vidal, Phys. Rev. B 70, 081402 (2004).

8. G. A. Wurtz, R. Pollard, and A. V. Zayats, Phys. Rev. Lett. 97, 057402 (2006).

9. C. Min, P. Wang, X. Jiao, Y. Deng, and H. Ming, Opt. Express 15, 12368 (2007).

10. C. Min, P. Wang, C. Chen, Y. Deng, Y. Lu, H. Ming, T. Ning, Y. Zhou, and G. Yang, Opt. Lett. 33, 869 (2008).

11. T. W. Ebbesen, H. L. Lezec, H. F. Ghaemi, T. Thio, and P. A. Wolff, Nature 391, 667 (1998).

12. A. Christ, S. G. Tikhodeev, N. A. Gippius, J. Kuhl, and H. Giessen, Phys. Rev. Lett. 91, 183901 (2003).

13. F. J. Garcia-Vidal, L. Martin-Moreno, T. W. Ebbesen, and L. Kuipers, Rev. Mod. Phys. 82, 729 (2010).

14. P. Ginzburg, A. Hayat, N. Berkovitch, and M. Orenstein, Opt. Lett. 35, 1551 (2010).

15. R. W. Boyd, Nonlinear Optics (Academic, 2003).

16. A. Taflove and S. Hagness Computational Electrodynamics: The Finite-Difference Time-Domain Method (Artech House, 2000).

17. P. Tran, Phys. Rev. B 52, 10673 (1995).

18. E. D. Palik, Handbook of Optical Constants of Solids II, E. D. Palik, ed. (Academic, 1991).

19. S. G. Rodrigo, F. J. García-Vidal, and L. Martín-Moreno, Phys. Rev. B 77, 075401 (2008).

20. V. Ta'eed, N. J. Baker, L. Fu, K. Finsterbusch, M. R. E. Lamont, D. J. Moss, H. C. Nguyen, B. J. Eggleton, D.-Y. Choi, S. Madden, and B. Luther-Davies, Opt. Express 15, 9205 (2007).

21. W. C. Hurlbut, Y.-S. Lee, K. L. Vodopyanov, P. S. Kuo, and M. M. Fejer, Opt. Lett. 32, 668 (2007).

22. L. Brzozowski, E. H. Sargent, A. S. Thorpe, and M. Extavour, Appl. Phys. Lett. 82, 4429 (2003). 